\title{
AN EXPEDITIOUS PARAMETRIC APPROACH FOR CITY INFORMATION MODELING AND FINITE ELEMENT ANALYSIS
}

\author{
F. M. La Russa ${ }^{1}$, M. Intelisano ${ }^{1}$, M. Galizia ${ }^{1}$, I. Caliò $^{1}$, C. Santagati ${ }^{1}$ \\ ${ }^{1}$ Department of Civil Engineering and Architecture, University of Catania \\ federico.larussa@phd.unict.it,imarco@hotmail.it,mgalizia@dau.unict.it, ivo.calio@unict.it, cettina.santagati@unict.it
}

\author{
Commission II
}

KEY WORDS: City Information Modeling, Computational Design, 3D city models, Seismic risk, Scan-to-FEM, VPL, Parametric modelling, 3D city models

\begin{abstract}
:
The mitigation of seismic risk passes through the assessment of seismic hazard of urban fabrics on a given territory. Statistical methods and damage probability matrices are currently used to facilitate seismic safety knowledge and assessment operations. These methods, despite being fast and low cost, often return results that differ from reality and prone to the expertise of the operator. Indeed, in order to have more accurate information it is necessary to conduct Finite Element Analysis (FEA). However, this type of analysis requires considerable surveying and modeling time and therefore are not easily applied to the urban scale. The key to implement this analysis at the territorial scale lies in the way of acquisition of urban data (geometric and informative) and their management within appropriate modeling environments that allow their treatment. Currently, the information modeling paradigms used for urban data collection and management are either time and resource consuming (HBIM) or overly simplified (GIS). In this research we investigate the potential of City Information Modeling (CIM) in a parametric environment (with reference to CityGML standards) combined with urban survey procedures. Aim of the work presented here is the definition of a parametric modeling protocol that allows, in a short time, the acquisition, modeling and finite element structural analysis of urban aggregates.
\end{abstract}

\section{INTRODUCTION}

The knowledge of the different degrees of seismic vulnerability of a territory allows to establish priorities of intervention in order to optimize the financial resources that local institutions can use for seismic risk mitigation. Therefore, it is necessary to establish priorities and to operate in terms of prevention with plans that aim at the progressive reduction of exposure.

Statistical methods and damage probability matrices are currently used to facilitate seismic safety knowledge and assessment operations. These methods, despite being fast and low cost, often return results that differ from reality and are prone to the expertise of the operator. Indeed, in order to have more accurate information it is necessary to conduct Finite Element Analysis (FEA) in digital environments. However, this type of analysis requires considerable surveying and modeling time and therefore are hardly applied to the urban scale. The key to implement this analysis at the urban scale lies in the way of acquisition of urban data (geometric and informative) and in their management within appropriate modeling environments that allow their treatment. In many regions exposed to seismic risk, the data needed to perform this type of analysis are often out of date, unusable, or absent (Geiß, 2015). In addition, traditional processes for acquiring this information, which include building-by-building inspections, are costly and time-consuming, making them impractical for evaluating a large building stock (Sulzer et al., 2018; Greco et al., 2020).

On the other hand, thanks to the evolution of geomatics methodologies several solutions are available today for acquiring large amounts of geometric data and generating complex models of real-world structures by exploiting point cloud processing. These procedures (known as Scan-to-FEM) are of support to define informative digital models useful for the purposes of modeling for FEA analysis. Although point cloud classification is largely optimized, automatic transition to useful informative digital models (BIM/GIS) for FEM analysis is currently a subject of research and in recent years parametric modeling is almost mainly used to solve problems related to interoperability with structural analysis software.

$\mathrm{n}$ this scenario, City Information Models which rely on BIM and GIS assets could represent the digital environment in which data useful for FEM analysis can be created, treated and exploited. The adoption of Computational Design (CD) into the field of 3D city models led to combining BIM and GIS procedures while minimizing input data and steps required towards the main purpose of the 3D city model requested. In addition, the granularity of the data allows for a high level of interoperability.

\subsection{Aim and structure of the paper}

In this research we investigate the potential of CIM in a parametric environment (with reference to CityGML standards) combined with urban survey procedures. Aim of the work presented here is the definition of a parametric modeling protocol that allows, in a short time, the acquisition, modeling and finite element structural analysis of urban aggregates. Our research questions are: is it possible to develop CIM models with expeditious urban survey procedures? Is it possible to obtain structural geometric models from a parametric CIM model to speed up the structural analysis?

Following a review of the state of art in Section 2, we describe the methodology adopted in Section 3. Section 4 focuses on the application of the procedure and its validation. In conclusion, in sections 5 and 6 we highlight the results of the research and indicate future developments.

\subsection{Application case}

With the purpose to test the entire workflow, we applied the procedure described to a one-store masonry building included in a historical city block in Catania (Italy). The construction under examination is included in a spontaneous building fabric of the the Santa Caterina al Rinazzo district. The variety of masonry building types present makes this block an exemplary case study 

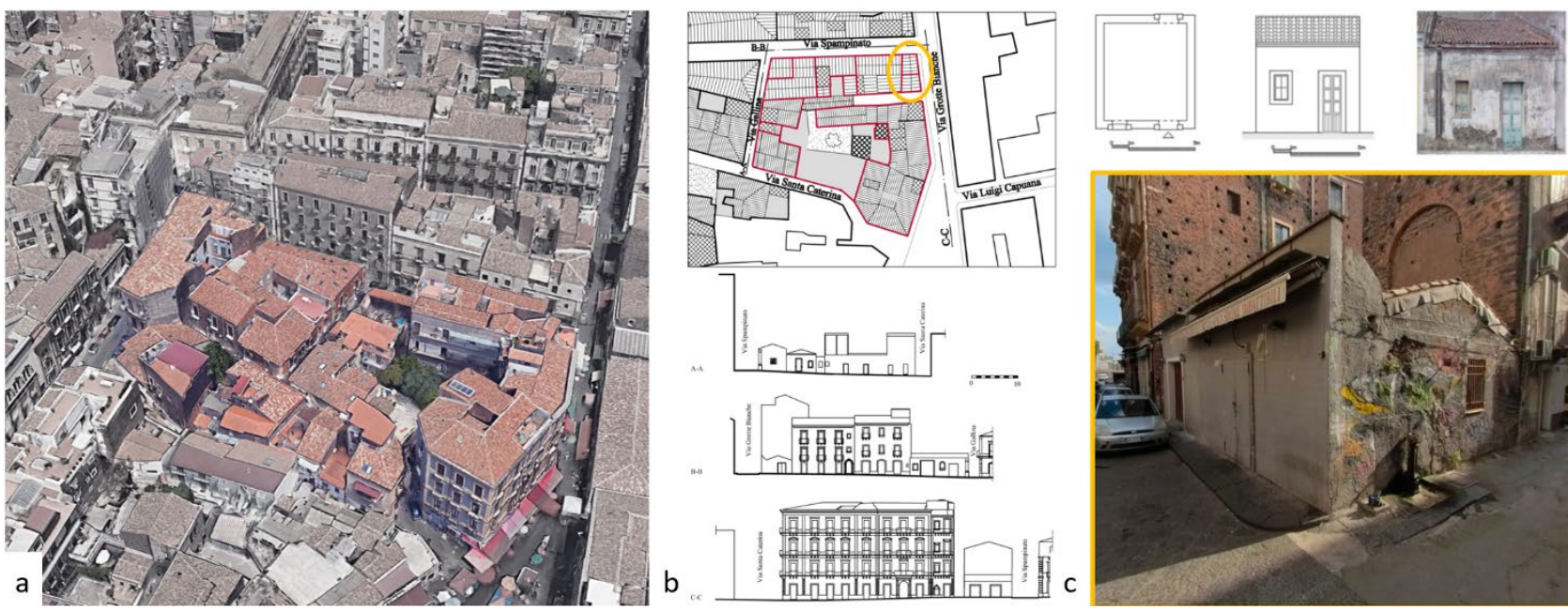

Figure 1. View of the block under analysis (a). Planimetry and sections of the block. Highlighted in yellow the ground floor house (b). Archetype of the ground floor house typology and photo of the case study (c).

of historic urban centers with high seismic vulnerability. In particular, the procedure suggested is applied to the one-storey building tipology (Fig. 1).

\section{RELATED WORKS}

This section first introduces and reviews City Information Modeling approaches (Section 2.1) and then discusses regards current Scan-to-FEM procedures (Section 2.2).

\subsection{City Information Modeling}

Defining City Information Modeling is not an easy task and it is currently the subject of considerable debate (Simonelli et al., 2018). According to one of its first definitions, a City Information Model consists of a system of urban elements represented by 2D and 3D elements containing information, linked by semantic relationships (Stojanovski, 2013). Therefore, we talk about City Information Modeling when we refer to the technologies and practices used to develop CIM models and to exploit their potential for urban and spatial analysis (Xue et al, 2021). We can think of CIM models as BIM systems applied to urbanism, a 3D semantic expansion of GIS models (Stojanovski, 2013). As Xu et al. highlight (2014), the major difficulty in CIM is the information modeling since these models deal with outdoor and indoor data semantically linked. It is worth to mention that $3 \mathrm{D}$ city models developed on BIM environments and then visualized in GIS (and vice versa) cannot fulfil the definition of CIM since geometries and information are not able to modify and interact to each other beyond visualisation and queries purposes. This type of 3D city models, also known as 'GeoBIM' (Noardo et al., 2020), is mainly focused on interoperability between BIM and GIS standard formats (IFC and CityGML) (Sun et al., 2020).

Nowadays, there is a wide range of CIM applications covering different disciplines (Xue et al., 2021; Xu et al., 2014). It is possible to distinguish three main approaches for developing CIM models: bottom-up, top-down and parametric.

The first one (or BIM-based) focuses more on remote sensing on site acquisition (close- and mid- range laser scanning and photogrammetry) with subsequent manual and semi-automatic modeling processes in BIM and CAD environments (Pelliccio et al., 2017; Zhang et al, 2021; Avena et al., 2021; Parrinello et al., 2020). These procedures often merge BIM and GIS data enabling the users to make queries and display models on web-based platform. In these models $\mathrm{CD}$ is applied, through Visual Programming Languages (VPL), to link, sort and merge metadata between models and environments but not for modeling purposes.

The top-down procedures deal mainly with long-range remote sensing techniques (e.g., Airborne LiDAR data) and geodata (coming from online open-data sources or datasets held by local institutions) which are further developed inside GIS-based procedural modeling digital environment (Biljecki et al, 2015; Nys et al., 2020; Wang et al., 2018, Pârvu et al., 2018). Top-down models usually don't need any further integration (unlike bottomup models) with exception for indoor data that are inserted via the conversion of IFC files into CityGML objects (Biljecki et al, 2021). These models are closer to the definition of CIM since they are based on CityGML standard where the city is treated as a whole system composed by different objects with geometries and metadata (OGC, 2020). In these models, CD is applied by using traditional textual programming languages for creating algorithms that, starting from point clouds segmentations, allow to obtain building geometries. The development paradigms for CIM presented so far are very expensive in terms of technologies and expertise needed. Therefore, they are not sustainable except for large cities, leaving small and medium centres excluded from the potential utility of CIM for emergency management.

In this context there is a third approach used for generating CIM model often called 'parametric urbanism' (De Jesus et al., 2018). This approach is characterized by CD workflows that often interoperate with open-data and remote sensing products. The main work environments are VPLs connected with CAD software. In particular, the VPL Grasshopper, thanks to several plugins dedicated to $3 \mathrm{D}$ city modeling, has supported the development of several research activities related to the CIM paradigm (De Jesus et al., 2018; Calvano et al., 2019; Fink \& Koenig, 2019; La Russa \& Genovese, 2021). The parametric approach relates to the previous ones regarding responsiveness between files of different nature (ex. IFC and SHP), interaction with digital survey products and standards for 3D city models (CityGML).

\subsection{Scan-to-FEM}

Concerning the transition from digital models to geometrical entities useful for FEA there are different approaches proposed in the last years. The evolution of geomatics methodologies allowed to acquire structures with high accuracy and to integrate in the same pipeline of work the application of BIM methodologies towards the definition of finite elements models. This procedure is known in literature as Scan-to-FEM (also 
Geodata DBs

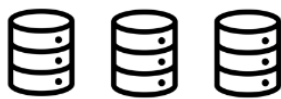

Visual surveys
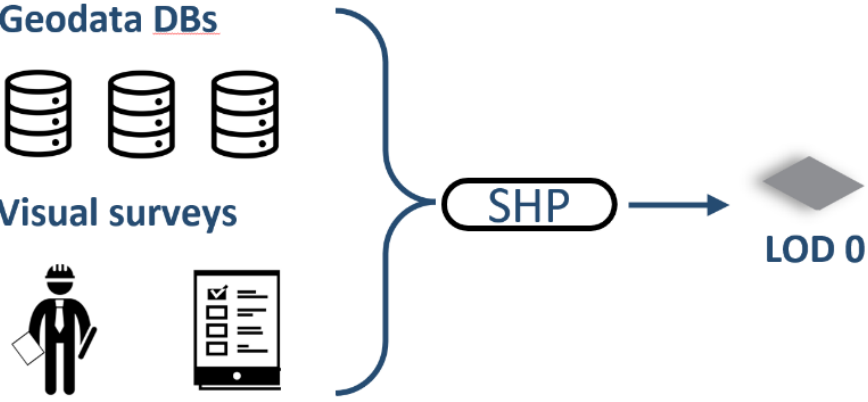

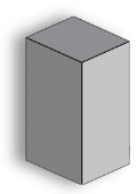

LOD 1

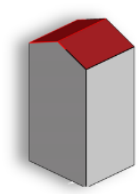

LOD 2

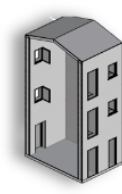

LOD 3

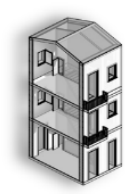

LOD 4

Figure 2. Conceptual scheme of the suggested procedure. The operator only needs to compile a shapefile.

Cloud-to-BIM-to-FEM) approach (Barazzetti et al., 2015; Dore et al., 2015). The transition from BIM to FEM contemplated in these methodologies does not always occur straightforwardly despite the interoperability of BIM models. Moreover, FEM analyses are not univocally defined by a single model, but they vary with regards to analysis needs (Abbate et al., 2020). Other methods do not take a BIM approach and move from point cloud to manual NURBS modeling. This procedure articulates in the following steps: generation of polygonal model from point cloud, extraction of outlines coming from the slices of the model, construction of NURBS model on the basis of sections and discretization of NURBS model into mesh model for analysis in dedicated FEM software (Fortunato et al., 2017). In the literature it is possible to find experiences of process automation that aim to save time and overcome the criticalities present in BIM models exported for FEM analysis (mesh compatibility, local deformations and too small details) (Castellazzi et al., 2015). It is worth to mention that there are not many applications towards the adoption of computational design tools for optimising and accelerating the Scan-to-FEM pipelines (Funari et al., 2021). Another research gap can be found in the lack of applications between city blocks acquisition-modeling and a semi-automatic transition to FEA.

\section{METHODOLOGY}

This section states the reasons and choices that led to the adoption of the parametric paradigm for the construction of a CIM for the purpose of seismic assessment at the urban scale via FEM analysis. The objective of this work is to develop a sustainable and expeditious methodology that allows professionals in any territory to have a simple but effective tool that leads to the development of functional CIMs for FEM analysis (Fig. 2). As described in Section 2, top-down and bottom-up CIM models require many resources for their development, in terms of technology and expertise. In the methodology descripted below, we decided to adopt the parametric paradigm because it allows to develop through VPL a code that is easier to manage for nonexperts (unlike top-down models) and that limits the effort of users to the acquisition of data in situ (unlike the tasks required in bottom-up models). The potential of the parametric paradigm lies in the automation and scalability of the process. It is possible to include algorithms that automatically optimize the transition to the polygonal model for FEM analysis. In addition, the versatility of the VPL code allows for the development and integration of solutions like bottom-up and top-down methods, thus ensuring interoperability (De Jesus et al., 2018). This section first describes the different steps of our framework (Section 3.1), then explains an expeditious and sustainable way to collect urban data (Section 3.2). Afterwards, it is outlined the structure of the VPL algorithm regarding modeling phases from LOD 0 to LOD 3 (Section 3.3) followed by the description of the discretization mechanism of the parametric model into polygonal model for FEM analysis (Section 3.4).

\subsection{Framework}

The proposed novel methodology can be summarized in the following main steps.

- Definition of a data acquisition protocol for parametric urban modeling;

- Development of VPL code for parametric modeling of data and geometries related to the urban area under analysis;

- Discretization of parametric information model into FEM geometric model and validation of the results.

At this stage of development, we considered tools and methods (from the acquisition of data, the development of the code to the creation of the structural model) widely known to professionals. QGIS and the OpenStreetMap portal (QGIS Development Team, 2021; OpenStreetMap Development Team, 2021) were used for the collection and consultation of urban data. Regarding the parametric modeling phase, we used the VPL Grasshopper $(\mathrm{GH})$ connected to the 3D modeling software Rhinoceros (Rhinoceros Development Team, 2021). Through the Grasshopper's plugins we created the code to export the geometry in several commercial software as SAP2000, FEM-Design, Karamba3D, OpenSees via Alpaca4D (SAP2000 Development Team, 2021; FEM-Design Development Team, 2021; Karamba3D, 2021; OpenSees Development Team, 2021; Alpaca4D, 2021).

\subsection{Urban data acquisition}

The urban data needed for modeling can be acquired from geodatabase which can be open (as in the case of OpenStreetMap) or held by local institutions. These sources usually provide the geometry of the footprints of the building associated with some attributes. Among these, we considered: number of elevations, average height of floors, building type, roof geometry, material types, etc. Table 1 lists the required data and their typology.

\begin{tabular}{|c|l|c|}
\hline Data requirements & \multicolumn{1}{|c|}{ Input data } & Type \\
\hline \multirow{4}{*}{ Basic Data } & Elevations & Integer \\
\cline { 2 - 3 } & Facade Orientation & Text \\
\cline { 2 - 3 } & Openings survey & Text \\
\cline { 2 - 3 } & Thickness survey & Text \\
\hline \multirow{4}{*}{ Typology Data* } & Roof type & Text \\
\cline { 2 - 3 } & Roof slope & Float \\
\cline { 2 - 3 } & Roof orientation & Text \\
\cline { 2 - 3 } & Interstorey & Float \\
\hline \multirow{2}{*}{ Deductible Data* } & Ridge height & Float \\
\cline { 2 - 3 } & Eaves height & Float \\
\hline
\end{tabular}

*These data are automatically replaceable by the GHPython component.

Table 1. Table listing the input data required by the user 


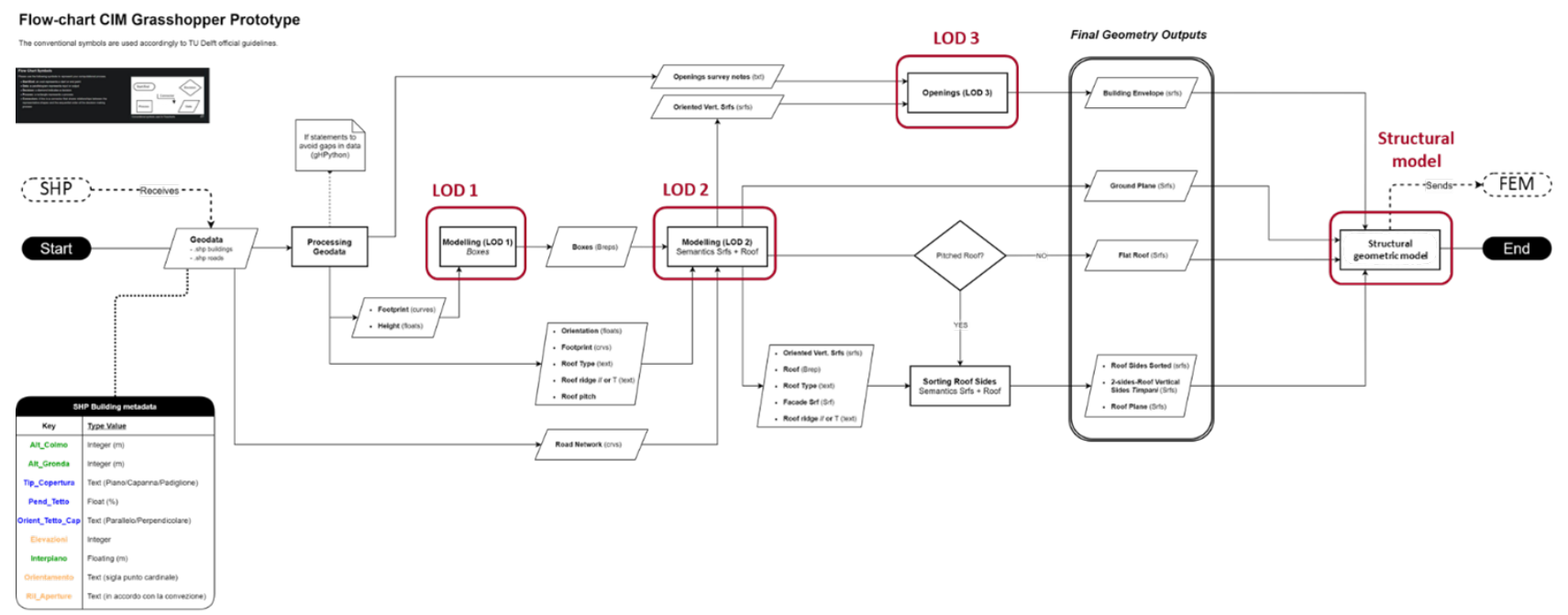

Figure 3. Flowchart of the VPL code in Grasshopper (TU Delft guidelines) for CIM modeling of the one-storey building typology.

In many cases, these data are not exhaustive of the geometrictechnological characteristics of the urban fabric under analysis, so a geometric or informative integration may be necessary. In case some data are not available, we have developed a GHPython component able to replace the missing data with data deducible from the inserted data or from the information related to the typical building typologies of the area. The development of the algorithm therefore contemplates a library of data related to techniques and materials typical of the analysis area.

The facade orientation (the polar direction of the surface normal of the facade) permits the definition of an order (counterclockwise) to univocally identify each vertical surface. This distinction permits the definition of a convention by which to describe the position of the openings on the different fronts and the thicknesses of the walls using a simple text string.

\subsection{From LOD 0 to LOD 4}

Once input data are collected, the GHPython component normalizes these ones for the implementation of the CIM model within GH. For the creation of the CIM, the CityGML international standard's sequence of levels of detail (LODs) is used as reference. Consequently, the following workflow occurs:

- LOD 0 (urban/landscape scale): it is a digital terrain model (DTM), on which an aerial photo is inserted, and which represents the topography of the area under study. Buildings are represented as a $2 \mathrm{D}$ ground footprint projected onto a $3 \mathrm{D}$ surface. In case the terrain morphology is not necessary for the objective of the model, buildings are represented by a horizontal plane for reducing the complexity of the model.

- LOD 1 (scale of the city or urban center): it consists in the representation of the buildings as bounding boxes; an indication of the height (as eaves height or number of floors) is required, thus delineating the volumetric relationships of the urban scene.

- LOD 2 (neighbourhood scale): compared to LOD 1, the geometry and the typology of the roofs are defined as well as the hierarchy of the surfaces in elevation (identification of the facade, of the remaining vertical surfaces and of the ground surface). The collected metadata are associated to the single surfaces thanks to the established convection.
- LOD 3 (architectural scale - exterior): openings are represented, and the overall detail is increased in exterior building components (e.g. roofs and the shapes of the openings).

- LOD 4 (architectural scale - interior): interior elements (floors, stairs, interior partitions, and doors) are added to the model; at this level the recognition of the building unit construction equipment is sufficiently detailed to complete the information enrichment of individual building units.

It is worth to mention that all the geometric entities can be represented by two-dimensional elements associated with attributes regarding thickness and other information. This choice allows to reduce the final weight and the management of the model. Indeed, beyond the purpose of visualisation, most of the time it is not necessary to represent $3 \mathrm{D}$ components for urban analysis (especially FEM analysis). However, the attributes inserted allow the possibility to include BIM modeling operations and interoperability thanks to external tools (e.g. VisualARQ, Rhino.Inside.Revit and Tekla-GH's connector) (VisualARQ Development Team, 2021; Rhino.Inside.Revit Development Team, 2021; Tekla Development Team, 2021).

\subsection{Parametric-to-FEM solutions}

Parametric modeling in the previous steps is based on NURBS modeling which is not sufficient for FEM analyses. Indeed, these ones require discrete polygonal models to deliver results (Barazzetti et al., 2015). Furthermore, when performing structural analysis, different software are used according to the accuracy and the results to be obtained. The parametric approach allows to develop different structural models from the same polygonal mesh thanks to numerous plugins released by software companies (see Section 3.1).

\section{EXPERIMENT AND RESULT}

In order to develop a prototype to test the methodology proposed in section 3 , we chose the one-level buildings included in a city block in the urban center of Catania (see section 1.2). The typology of this building, called casa terrana ('ground floor house'), can be fully described for CIM purposes by reaching LOD 3 (Fig. 3). For this building typology, LOD 3 and LOD 4 coincide. 

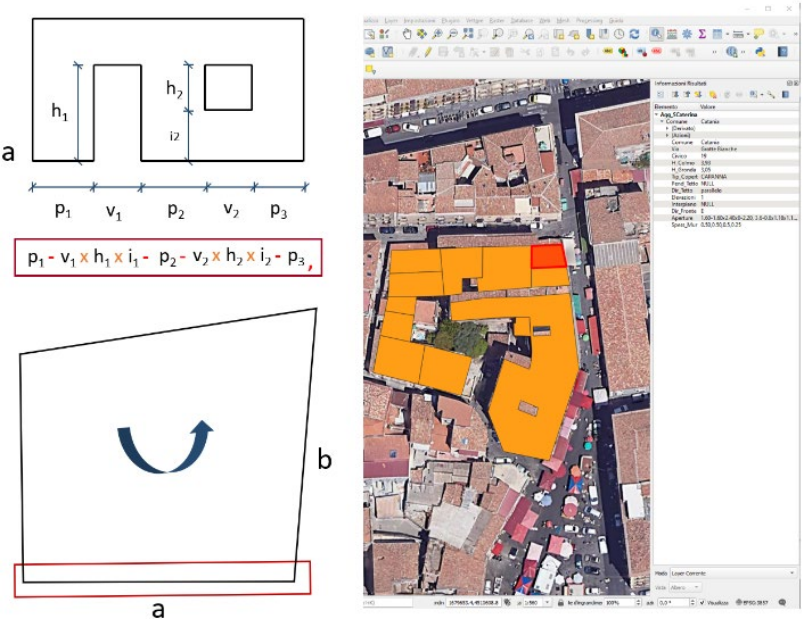

Figure 4. From left, convention for openings and shape file.

\subsection{Parametric CIM modeling LOD 0 to LOD 3}

The building chosen as a case study was included in a mapping process that produced geodata on the historic center. These geodata consist of a shapefile that, in addition to the building footprints, contains various metadata about each building (Galizia \& Santagati, 2012). Through the Urban GH plugin, it was possible to read inside $\mathrm{GH}$ both the geometries and the metadata contained in the shapefile (Fig. 4). The initial modeling step was to achieve LOD 1 (box) according to the standards defined by CityGML. Afterwards, LOD 2 was reached by deconstructing the box from LOD 1 and semantically identifying the surfaces by taking the surface normals and coordinates of the barycentres as references. Finally, for LOD 3 the openings were inserted by extracting position and size from the text strings initially inserted (Fig 5).

LOD 1

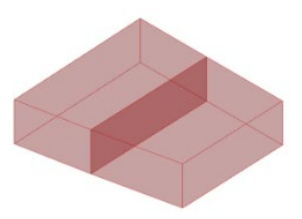

- Volumes definition

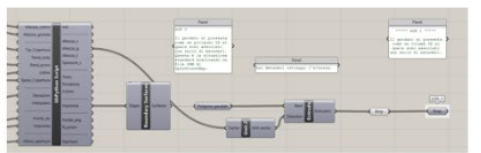

LOD 2

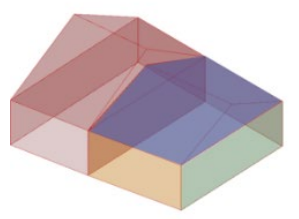

- Roof geometries

- Semantic classification of sufaces

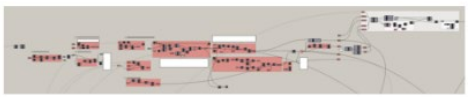

LOD 3

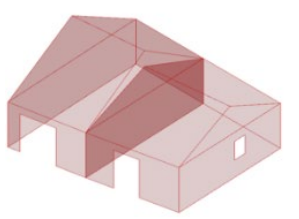

\section{- Openings placement}

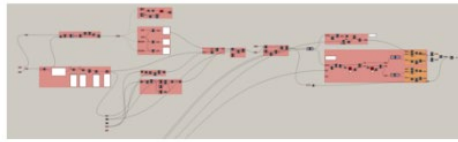

Figure 5. Parametric CIM modeling phases (LOD $0-3$ )

\subsection{Parametric-to-FEM automatic discretization}

With the aim to make the mesh of the FEM model seamless, all NURBS surfaces were treated using GH tools for meshmanipulation that allowed to preserve the conformity of the mesh between the surfaces of the model. Finally, the mesh model has been used for FEM analysis through several plugins for $\mathrm{GH}$ related to structural analysis software. Some of the plugins made it possible to perform structural analysis directly within the Grasshopper workspace. This is the case for Karamba3D and Alpaca4D (OpenSees). Other ones have worked as connectors transporting (automatically) the mesh model from Grasshopper to the working environment of the analysis software. In particular, for SAP2000 we developed a specific component that enables the user to extract the mesh model from $\mathrm{GH}$ and to import it into SAP2000 (Fig. 6).

Grasshopper

(Rhinoceros) Mesh Model for FEM Analysis
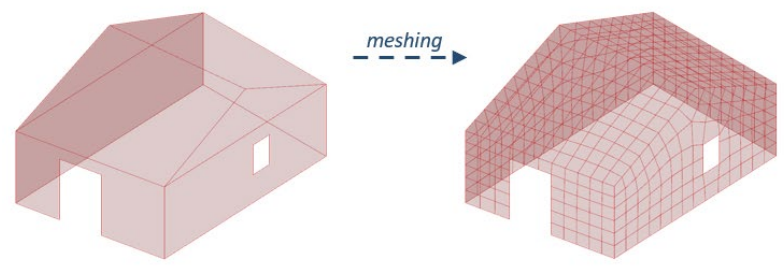

FEM Models
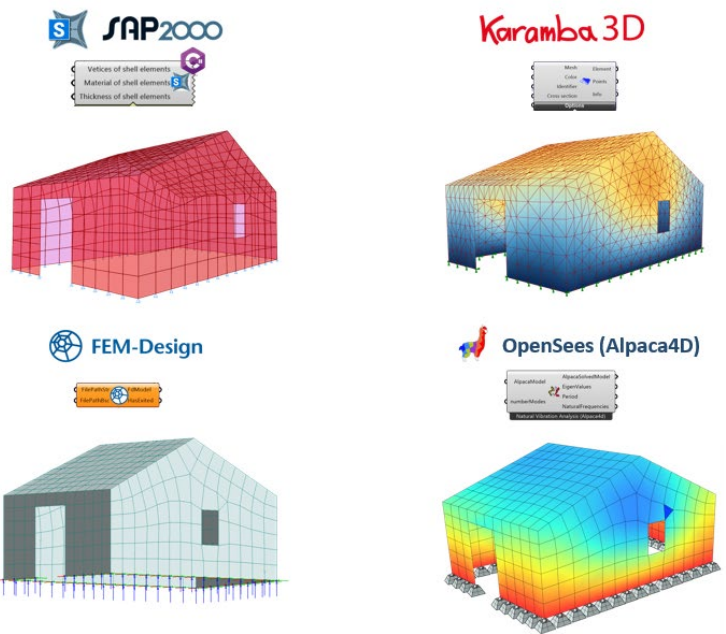

Figure 6. Parametric CIM model discretized into FEM models

\subsection{Validation of FEM Models obtained and comparison with traditional procedure}

To verify the procedure and the results that can be extracted from the analyses performed on these models, we proceeded with an analysis of the vibration periods with the same system configuration (geometric and material). In Figure 8 are reported results and deformations obtained.

The results are homogeneous in respect of the tolerances due to the intrinsic differences of the calculation software used. Moreover, from the qualitative analysis of the deformations obtained, the behaviour of the geometries is consistent. In conclusion, it has been made a comparison of the modeling times 


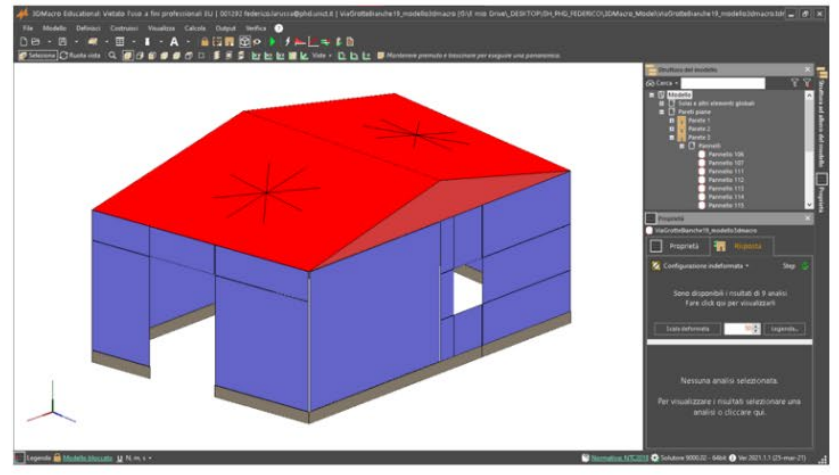

Manual modeling on 3DMacro

- DXF creation for reference lines $30^{\prime}$ 1h $40^{\prime}$ $20^{\prime}$

2h 30'
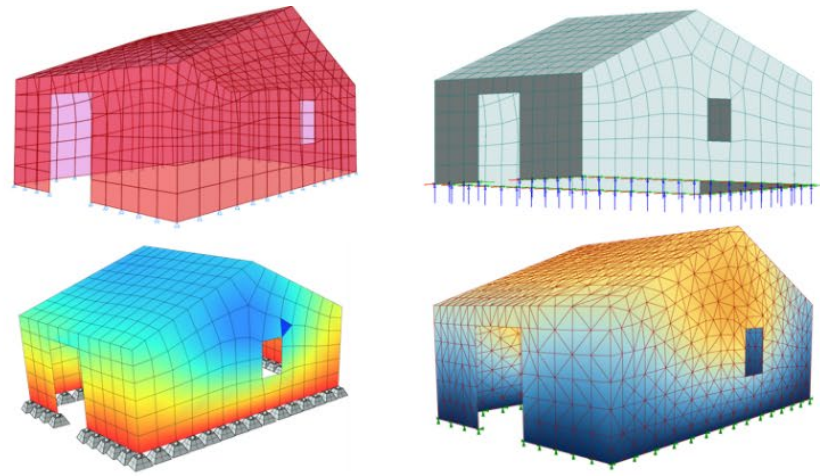

\section{CIM parametric modeling}

- Inserting input data

$10^{\prime} 0^{\prime \prime}$

- CIM computational time

$0^{\prime} 12^{\prime \prime}$

- FEM model generation

0' 7'

Total

$10^{\prime} 19^{\prime \prime}$

\section{$\mathrm{N}^{\circ}$ of models}

$1 \quad N^{\circ}$ of models

Figure 7. Comparison of modeling times between a traditional FEM modeling procedure and parametric CIM modeling.

taking as reference the same geometry but modelled inside a software (well-known and diffused among professionals) for structural analysis of masonry buildings (3DMacro Development Team, 2021). In figure 7 are shown models and times of comparison.

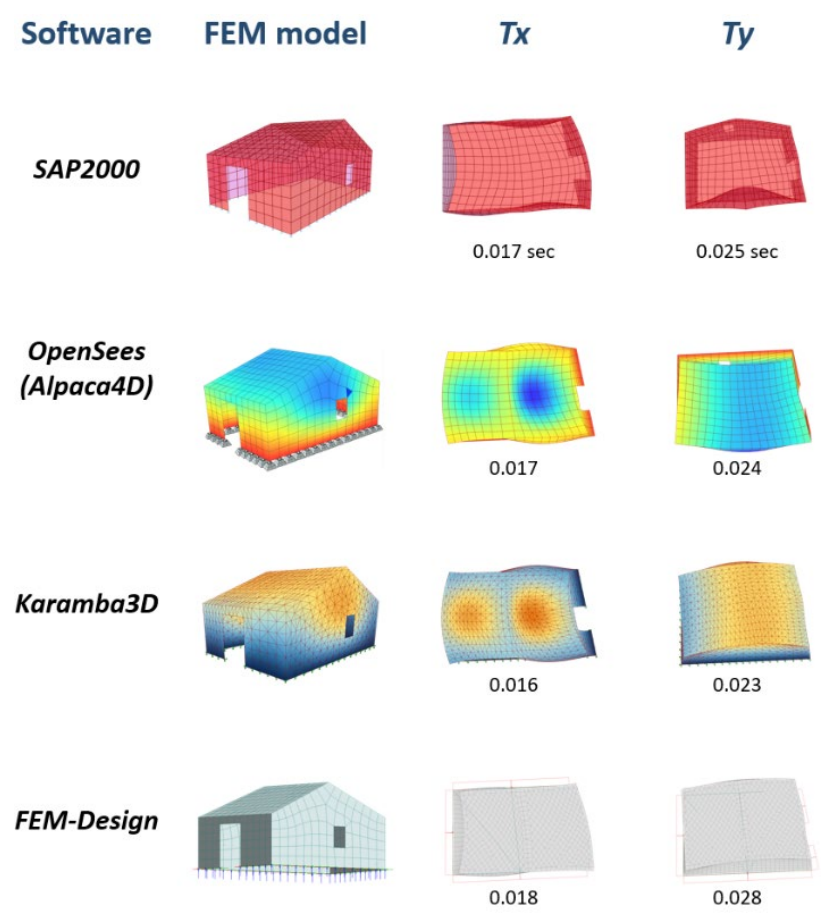

Figure 8. Analysis of the vibration periods of the same model.

\section{DISCUSSION}

The developed framework allows to state that it is possible to develop a CIM model in an expeditious way aimed at seismic vulnerability analysis. The VPL code can be reused for modeling and the user has only the task of data collection. Therefore, this methodology may be feasible in the case of crowdmapping campaigns. An important result is the responsiveness of the parametric approach, in fact unlike bottom-up methods (see Section 2.1) the automation of most of the modeling processes allows to define and update the model in a short time also by not high specialized operators. In this direction, it is important to highlight the possibility of implementing the framework by automating the acquisition of some input data (such as the survey of openings and the type of roof) through the adoption of advanced remote sensing techniques (Grilli et al., 2021; La Russa et al., 2021). Another remarkable outcome is that the geometric structural model obtained is univocally defined, regardless of the operator who generates it. Therefore, any possible mistake due to a distraction, a different way of conceiving the structure, and/or modeling phases it is reduced. Of course, the operator can after modify and integrate the model according to his needs.

\section{CONCLUSION AND FUTURE WORKS}

In this paper we tested the potential of the parametric paradigm for the development of City Information Models in an expeditious and sustainable manner with the goal of facilitating seismic vulnerability analysis at the urban scale. The results of this research activity consist in the development of a VPL code that allows to create a CIM model up to LOD 3 and in the use of parametric modeling to obtain mesh models for FEM analysis. The next steps in the research aim to further develop the potential that has emerged from the parametric approach to CIM modeling. In particular, we will investigate: the issue of internal partitions (corresponding to LOD 4) in the absence of surveys for the purpose of seismic risk, and the interoperability of parametric CIM models through the definition of a CityJSON models from parametric models. 


\section{AUTHOR CONTRIBUTIONS}

Conceptualization: C.S, M.G., I.C., F.M.L.R.; Methodology: C.S, M.G., I.C., F.M.L.R., M. I.; Investigation: F.M.L.R.; Software: F.M.L.R., M. I.; Validation: F.M.L.R., M.I,; Writing F.M.L.R.; Visualization: F.M.L.R.; Supervision: C.S.

\section{REFERENCES}

Abbate, E., Invernizzi S., Spanò, A., 2020. HBIM parametric modeling from clouds to perform structural analyses based on finite elements: a case study on a parabolic concrete vault. Applied Geomatics. 10.1007/s12518-020-00341-4

Alpaca4d (OpenSees) Pellegrino M. and Gaudioso D., 2021. GH Plugin for using OpenSees inside Grasshopper, Version 0.0.5 . food4rhino.com/en/app/alpaca4d (15 January 2022).

Avena M., Colucci E., Sammartano G., Spanò A., 2021. HBIM MODELING FOR AN HISTORICAL URBAN CENTRE. The International Archives of the Photogrammetry, Remote Sensing and Spatial Information Sciences. XLIII-B2-2021. 831-838. 10.5194/isprs-archives-XLIII-B2-2021-831-2021.

Barazzetti L., Banfi F., Brumana R., Gusmeroli G., Previtali M., Schiantarelli G., 2015. Cloud-to-BIM-to-FEM: Structural simulation with accurate historic BIM from laser scans, Simulation Modeling Practice and Theory, Volume 57, 2015, 7187, ISSN

$1569-190 X$

https://doi.org/10.1016/j.simpat.2015.06.004

Biljecki, F., Stoter, J., Ledoux, H., Zlatanova, S., Çöltekin, A., 2015. Applications of 3D City Models: State of the Art Review. ISPRS Int. J. Geo-Inf. 2015, 4, 2842-2889. https://doi.org/10.3390/ijgi4042842

Biljecki, F., Lim, J., Crawford, J., Moraru, D., Tauscher, H., Konde, A., Adouane, K., Lawrence, S., Janssen P., Stouffs, R., 2021. Extending CityGML for IFC-sourced 3D city models. Automation in Construction. 121. 103440. 10.1016/j.autcon.2020.103440.

Calvano, M., Empler, T., Caldarone A., 2019. L'ARIM per la prevenzione del rischio sismico. Disegnare Idee Immagini, 59, 70-81.

Castellazzi, G., D'Altri, A., Bitelli, G., Selvaggi, I., Lambertini, A., 2015. From Laser Scanning to Finite Element Analysis of Complex Buildings by Using a Semi-Automatic Procedure. Sensors. 15. 18360. 10.3390/s150818360.

De Jesus, E., Amorim, A., Groetelaars, N., Fernandes, V., 2018. MODELING CITIES FOR 3D GIS PURPOSES. ISPRS International Archives of the Photogrammetry, Remote Sensing and Spatial Information Sciences. XLII-4. 135-142. 10.5194/isprs-archives-XLII-4-135-2018.

Dore, C., Murphy, M., McCarthy, S., Brechin, F., Casidy, C., Dirix, E., 2015. Structural Simulations and Conservation Analysis -Historic Building Information Model (HBIM). ISPRS - International Archives of the Photogrammetry, Remote Sensing and Spatial Information Sciences. XL-5/W4. 351-357. 10.5194/isprsarchives-XL-5-W4-351-2015

FEM-Design Development Team, 2021. FEM-Design, Version 20.00.005. StruSoft. strusoft.com/products/fem-design (15 January 2022).
Fink, T., Koenig, R., 2019. Integrated Parametric Urban Design in Grasshopper / Rhinoceros 3D: Demonstrated on a Master Plan in Vienna. Proceeding of eCAADe SIGraDi 2019, Volume 1 10.5151/proceedings-ecaadesigradi2019 398.

Fortunato, G., Funari, M., Lonetti, P., 2017. Survey and seismic vulnerability assessment of the Baptistery of San Giovanni in Tumba (Italy). Journal of Cultural Heritage. 26. 64-78. 10.1016/j.culher.2017.01.010.

Funari, M., F., Hajjat, A., E., Masciotta, M., G., Oliveira, D., V., 2021. A Parametric Scan-to-FEM Framework for the Digital Twin Generation of Historic Masonry Structures. Sustainability 2021, 13, 11088. https://doi.org/10.3390/su131911088

Galizia, M., Santagati, C., 2012. GIS is/and knowledge. Documenting and managing Catania's historic urban heritage, Journal of Civil Engineering and Architecture, Volume 6 (8), 1014-1020. DOI:10.17265/1934-7359/2012.08.009

Geiß, C., 2015. Seismic vulnerability assessment of built environments with remote sensing. PhD Dissertation, , HumboldtUniversität zu Berlin, http://dx.doi.org/10.18452/17104

Greco, A., Lombardo, G., Pantò, B. \& Famà, A., 2020. Seismic Vulnerability of Historical Masonry Aggregate Buildings in Oriental Sicily, International Journal of Architectural Heritage, 14:4, 517-540, DOI: 10.1080/15583058.2018.1553075

Grilli, E., Poux, F., Remondino, F., 2021. UNSUPERVISED OBJECT-BASED CLUSTERING IN SUPPORT OF SUPERVISED POINT-BASED 3D POINT CLOUD CLASSIFICATION. The International Archives of the Photogrammetry, Remote Sensing and Spatial Information Sciences. XLIII-B2-2021. 471-478. 10.5194/isprs-archivesXLIII-B2-2021-471-2021.

Karamba3D (GH plugin) - Preisinger, C. (2013), Linking Structure and Parametric Geometry. Architectural Design, 83: 110-113 DOI: 10.1002/ad.1564.

La Russa, F., M., Genovese, G., 2021. City Information Modeling and seismic risk: integrated HBIM-GIS approaches for the management of post-earthquake scenarios. In: (eds.) Cecilia Bolognesi e Cettina Santagati, Dn - Building Information Modeling, Data \& Semantics, 8, 72 - 83, ISSN 2610-8755

La Russa, F., M., Galizia, M., Santagati, C., 2021. REMOTE SENSING AND CITY INFORMATION MODELING FOR REVEALING THE COMPLEXITY OF HISTORICAL CENTERS, Int. Arch. Photogramm. Remote Sens. Spatial Inf. Sci., XLVI-M-1-2021, 367-374, https://doi.org/10.5194/isprsarchives-XLVI-M-1-2021-367-2021, 2021.

Noardo, F., Harrie, L., Arroyo Ohori, K., Biljecki, F., Ellul, C., Krijnen, T., Eriksson, H., Guler, D., Hintz, D., Jadidi, M., A., Pla, M., Sanchez, S., Soini, V.-P., Stouffs, R., Tekavec, J., Stoter, J., 2020. Tools for BIM-GIS Integration (IFC Georeferencing and Conversions): Results from the GeoBIM Benchmark 2019. $\begin{array}{llllll}\text { ISPRS Int. J. Geo-Inf. 2020, 9, } 502 . & \text {. }\end{array}$ https://doi.org/10.3390/ijgi9090502

Nys, G., A., Poux, F., Billen, R., 2020. CityJSON Building Generation from Airborne LiDAR 3D Point Clouds. ISPRS International Journal of Geo-Information. 9. 521. 10.3390/ijgi9090521. 
OGC, CityGML 3.0 Conceptual Model, (2020). Link: https://github.com/opengeospatial/CityGML-3.0CM Last access on: $20 / 01 / 2022$

OpenSees Development Team, 2021. Open System for Earthquake Engineering Simulation (OpenSees),Version 3.3.0 . Open Source project leaded by University of California (Berkeley). opensees.berkeley.edu (15 January 2022).

OpenStreetMap Development Team, 2021. Open Source project. OpenStreetMap Community. openstreetmap.org (15 January 2021)

QGIS Development Team, 2021. Quantum GIS, Version 3.12. Open Source project. qgis.org (15 January 2021).

Parrinello, S., De Marco, R., Galasso, F., 2020. An urban modeling protocol through catalogues and technological modules. From digital survey to the 3D information system for the historic center of Bethlehem. Dn - Building Information Modeling, Data \& Semantics, 6, 52 - 69.

Pârvu, I., Remondino, F., Ozdemir, E., 2018. LOD2 Building Generation Experiences and Comparisons. Journal of Applied Engineering Sciences, 8(2) 59-64. https://doi.org/10.2478/jaes2018-0019

Pelliccio, A., Saccucci, M., Grande, E., 2017. HT_BIM: Parametric modeling for the assessment of risk in historic centers. In DISEGNARECON, 10/18, pp. 5.1 - 5.12

Pereira, A., Buzzo, M., Zimermann, I., Neto, F., Malgarezi, H., 2021. A Descriptive 3D City Information Model Built From Infrastructure BIM: Capacity Building as a Strategy for Implementation. International Journal of E-Planning Research. 10. 1-14. 10.4018/IJEPR.20211001.oa9.

Rhino.Inside.Revit Development Teams, 2021. Robert McNeel \& Associates, Version 2021. rhino3d.com/it/features/rhinoinside-revit/ (13 January 2022).

Rhinoceros Development Team, 2020. Rhinoceros and Grasshopper, Version 7. Robert McNeel \& Associates. rhino3d.com (15 January 2022).

SAP2000 Development Team, 2021. Structural Analysis Program (SAP) 2000, Version 23.3.1 Computers and Structures Inc. (CSI). csi-italia.eu/software/sap2000/ (15 January 2022).

Stojanovski, T., 2013. City information modeling (CIM) and urbanism: Blocks, connections, territories, people and situations. Simulation Series. 45. 86-93.

Simonelli, L., Amorim, A., L., d., 2018. City Information Modeling: General Aspects and Conceptualization. American Journal of Engineering Research, 7(10), 319-324

Sulzer, R., Nourian, P., Palmieri, M., Gemert, J., 2018. SHAPE BASED CLASSIFICATION OF SEISMIC BUILDING STRUCTURAL TYPES. ISPRS - International Archives of the Photogrammetry, Remote Sensing and Spatial Information Sciences. XLII-4/W10. 179-186. 10.5194/isprs-archives-XLII4-W10-179-2018

Sun, J., Olsson, P., Eriksson, H., Harrie, L., 2020. Evaluating the geometric aspects of integrating BIM data into city models. Journal of Spatial Science, 65(2), 235-255
Tekla Development Team, 2021. Version 2021. Trimble Navigation. tekla.com (13 January 2022).

VisualARQ Development Team, 2021. Asuni CAD S. A. visualarq.com (13 January 2022).

Wang, R., Peethambaran, J., Dong, C., 2018. LiDAR Point Clouds to 3D Urban Models: A Review. IEEE Journal of Selected Topics in Applied Earth Observations and Remote Sensing. PP. 10.1109/JSTARS.2017.2781132.

Xue, F., Wu, L., Lu, W., 2021. Semantic enrichment of Building and City Information Models: a ten-year review. In Advanced Engineering Informatics, 47, 101245. DOI: 10.1016/j.aei.2020.101245

Xu, X., Ding, L., Luo, H., Ma, L., 2014. From building information modeling to city information modeling. In ITcon Vol. 19, Special issue BIM Cloud-Based Technology in the AEC Sector: Present Status and Future Trends, 292 - 307. Link: https://www.itcon.org/2014/17

Zhang, H., Yuan, X., Yang, X., Han, Q., Wen, Y., 2021. The integration and application of BIM and GIS in modeling. Journal of Physics: Conference Series, 1903(1), 012074. https://doi.org/10.1088/1742-6596/1903/1/012074

3DMacro Development Team, 2021. 3DMacro. Software for Masonry Buildings, Version 2021.1.1. Gruppo Sismica S.r.1. murature.com/software.php?id=1\&n=3DMacro $\quad(15$ January 2022) 